\title{
Creation and detection of a mesoscopic gas in a non-local quantum superposition
}

\author{
Christoph Weiss and Yvan Castin \\ Laboratoire Kastler Brossel, École Normale Supérieure, \\ UPMC and CNRS, 24 rue Lhomond, 75231 Paris Cedex 05, France
}

(Dated: October 24, 2018)

\begin{abstract}
We investigate the scattering of a quantum matter wave soliton on a barrier in a one dimensional geometry and we show that it can lead to mesoscopic Schrödinger cat states, where the atomic gas is in a coherent superposition of being in the half-space to the left of the barrier and being in the half-space to the right of the barrier. We propose an interferometric method to reveal the coherent nature of this superposition and we discuss in details the experimental feasibility.

PACS numbers: 03.75.Gg, 03.75.Lm, 34.50.-s
\end{abstract}

It is now possible to control the strength of the atomic interaction in a gas, with Feshbach resonances. This has allowed the observation of single matter wave bright solitons with thousands of atoms [1] or a train of solitons [2] with ${ }^{7} \mathrm{Li}$ atoms trapped in a one-dimensional (1D) geometry. These solitons are quantum bound states of a mesoscopic gas, which opens up fascinating possibilities: Apart from testing mean field predictions in these systems [3], one can address truly quantum problems, issuing from the quantum nature of the gas center of mass.

In particular, it was recently proposed to use a BoseEinstein condensate in interferometric experiments to test the existence of decoherence mechanisms not predicted by usual quantum mechanics and that would show up for very massive particles [4]. Experiments have succeeded in observing interferences with molecules as big as fullerenes and there is a need for more massive interferometric objects [5]. A soliton with a small number of 100 ${ }^{7} \mathrm{Li}$ atoms has the same mass as $\mathrm{C}_{60}$, with appealing new features: It does not have internal bound states other than its ground state, it can be reversibly dissociated in an unbound atomic gas via a Feshbach resonance, and it allows the exploration of a new regime, in which the center of mass kinetic energy of the interfering object is of the same order as the binding energy of its constituents.

Furthermore, thanks to the extremely low temperatures accessible in atomic gases, down to $0.45 \mathrm{nK}$ 6], and the weak decoherence present in these systems 7], one may hope to split the center of mass wavefunction of the solitonic gas in two wavepackets that would keep their mutual coherence over mesoscopic distances, say a fraction of a millimeter, much larger than the size of the soliton. The gas would then have simultaneously non-zero probability amplitudes of being in two different spatial locations, thus forming a mesoscopic Schrödinger cat in real space. One may then ascertain the presence of a cat state by recombining and interfering these two mesoscopically different quantum states of the gas. This would constitute a generalization to many atoms of the oneion experiment of [8]. While mesoscopic Schrödinger cat states have been reported for radiation fields [9] they have not been reported yet with ultracold atoms, and atom op- tics with a quantum soliton is a promising alternative to existing ideas for cat production in these systems [10].

The dynamics of the center of mass wavepacket during the scattering of the soliton on a barrier raises non trivial theoretical issues, since the presence of the barrier makes the 1D many-body problem non integrable via the Bethe Ansatz. We thus construct an approximate effective lowenergy Hamiltonian for the center of mass of the gas, and we derive a rigorous upper bound on the resulting error.

The starting point is the many-body Hamiltonian in 1D, for $N$ bosonic particles of mass $m$ interacting via the usual contact interaction of coupling constant $g$, in presence of the barrier potential $U(x) \geq 0$ :

$$
H=\sum_{i=1}^{N}\left[\frac{p_{i}^{2}}{2 m}+U\left(x_{i}\right)\right]+g \sum_{i<j} \delta\left(x_{i}-x_{j}\right) .
$$

This is conveniently rewritten as $H=P^{2} /(2 M)+H_{\text {in }}+V$, singling out the kinetic energy of the center of mass ( $M=N m$ is the total mass and $P$ the total momentum of the gas), the so-called internal Hamiltonian $H_{\text {in }}$ and the sum of the $N$ barrier potentials, $V$. Without a barrier $(V \equiv 0)$ there is full separability between the center of mass and the internal variables, so that we split the Hilbert space as a tensorial product of center of mass and internal variables. $H_{\text {in }}$ is diagonalized with the Bethe ansatz [1]]: For $N$ fixed, its ground state is its single discrete eigenstate, the quantum soliton $|\phi\rangle$ of energy $E_{0}(N)$ [12], separated from a continuum of solitonic fragments by an energy gap which is minus the chemical potential,

$$
|\mu|=E_{0}(N-1)-E_{0}(N)=\frac{m g^{2} N(N-1)}{8 \hbar^{2}} .
$$

In presence of a barrier, we consider the scattering state $|\Psi\rangle$ of the soliton with an incoming center of mass wavevector $K>0$. We restrict to a low incoming kinetic energy to have elastic scattering,

$$
E-E_{0} \equiv \frac{\hbar^{2} K^{2}}{2 M}<|\mu|
$$

Far from the barrier, one can then observe only a nonfragmented soliton, to the right with the transmission amplitude $t$, to the left with the reflection amplitude $r$. 
In this elastic regime, an effective hermitian Hamiltonian may be defined, introducing the projector $\mathcal{P}=I_{\mathrm{CoM}} \otimes$ $|\phi\rangle\langle\phi|$ acting as the identity on the center of mass and projecting the internal state on its ground state, so that

$$
\mathcal{P}|\Psi\rangle=|\Phi\rangle \otimes|\phi\rangle .
$$

Far from the barrier, $\Phi(X)$ is simply the center of mass wavefunction, $X$ being the center of mass position. The so-called $\mathcal{P} G \mathcal{P}$ formalism, where $G$ is the resolvent of the full Hamiltonian [13], then gives the exact equation

$$
\frac{\hbar^{2} K^{2}}{2 M}|\Phi\rangle=\left[\frac{P^{2}}{2 M}+\bar{V}(X)+\delta V\right]|\Phi\rangle .
$$

The first contribution to the effective potential, in the right hand side of (5), is the convolution of the barrier potential with the internal density profile of the soliton:

$$
\bar{V}(X)=\langle\phi|V| \phi\rangle=\int_{-\infty}^{+\infty} d x U(X-x) \rho(x \mid 0)
$$

where $\rho(x \mid 0)$ is the mean density of particles in the soliton knowing that the center of mass is localized in $X=0$. It was calculated with the Bethe ansatz [14] and is well approximated for $N \gg 1$ by the mean field density profile $\rho(x \mid 0) \simeq N /\left[4 \xi \cosh ^{2}(x / 2 \xi)\right]$, where the mean field soliton size is $\xi=\hbar^{2} /(m|g| N)$. The second contribution in (5) involves virtual transitions to internal excited states:

$$
\delta V=\left\langle\phi\left|V \mathcal{Q} \frac{\mathcal{Q}}{E \mathcal{Q}-\mathcal{Q} H \mathcal{Q}} \mathcal{Q} V\right| \phi\right\rangle
$$

where $\mathcal{Q}=I-\mathcal{P}$. We shall neglect this contribution but not without a justification. From the fact that $\mathcal{Q} H \mathcal{Q} \geq$ $E_{0}+|\mu|$, a consequence of the positivity of $P^{2} / 2 M$ and $V$, and of the energy gap of $H_{\text {in }}$, we see in the regime (3) that the operator $-\delta V$ is positive and bounded as

$$
-\delta V \leq W(X) \equiv \frac{\left\langle\phi\left|V^{2}\right| \phi\right\rangle-\bar{V}(X)^{2}}{|\mu|-\hbar^{2} K^{2} / 2 M} .
$$

When one neglects $\delta V$ in (5), the exact $\Phi(X)$ is replaced by $\Phi_{0}(X)$, which involves the same incoming wave $e^{i K X}$, but with outgoing waves $e^{i K|X|}$ whose transmission and reflection amplitudes $t_{0}$ and $r_{0}$ are only approximate. We have rigorously bounded the resulting errors. We discuss here only the experimentally relevant case of an even barrier $U(x)=U(-x)$. Introducing the "small parameter", $\epsilon \equiv M\left\langle\Phi_{0}|W(X)| \Phi_{0}\right\rangle /\left(\hbar^{2} K\left|t_{0}\right|\right)$, we have for $\epsilon<1 / 2$ the following theorem:

$$
\left|t-t_{0}\right| \text { and }\left|r-r_{0}\right| \leq \frac{\left|t_{0}\right| \epsilon}{1-2 \epsilon} .
$$

It remains to calculate $W(X)$. We have derived from the Bethe ansatz the large $N$ asymptotic expression [15]

$$
\begin{aligned}
W(X) & \simeq \frac{2 N \xi^{4}}{|\mu|-\frac{\hbar^{2} K^{2}}{2 M}} \int_{-\infty}^{+\infty} d x \int_{x}^{+\infty} d y U^{\prime \prime}(X+x \xi) \\
& \times U^{\prime \prime}(X+y \xi) \frac{2+y-x}{\left(e^{y}+1\right)\left(e^{-x}+1\right)} .
\end{aligned}
$$

In practice, the barrier $U(x)$ is produced with a Gaussian laser beam, $U(x)=U_{0} \exp \left(-2 x^{2} / b^{2}\right)$, with a waist $b$ much larger than the soliton size $\xi$. Then the mean potential $\bar{V}(X)$ is close to $N U(X)$. We shall also assume that the incoming kinetic energy $\hbar^{2} K^{2} / 2 M$ is about half the gap $|\mu| \simeq \hbar^{2} / 8 m \xi^{2}$, so that (3) is satisfied without paying the price of very slow soliton velocities. Then $K b \gg 1$ and the scattering is in the semi-classical regime [17], where approximate expressions can be obtained for $t_{0}$ and $r_{0}$. A transmission probability $1 / 2$ is predicted to be achieved for an incident wavevector $K_{0}$ such that

$$
\frac{\hbar^{2} K_{0}^{2}}{2 M}=\max _{X} \bar{V}(X) \simeq N U_{0} .
$$

In the vicinity of $K=K_{0}$, the transmission probability varies sharply from zero to unity,

$$
\left|t_{0}\right|^{2} \simeq \frac{1}{1+\exp \left[\frac{K_{0}-K}{\delta K}\right]} \quad \text { with } \delta K \simeq \frac{1}{\pi \sqrt{2} b} .
$$

It remains to estimate the bound (9). One may take $U^{\prime \prime} \simeq U^{\prime \prime}(X)$ in (10), since $b \gg \xi$, so that

$$
W(X) \simeq \frac{N \xi^{4}}{|\mu|-\frac{\hbar^{2} K^{2}}{2 M}}\left[U^{\prime \prime}(X)\right]^{2}\left[\frac{2 \pi^{2}}{3}+4 \zeta(3)\right] .
$$

In $K=K_{0}$, for $\epsilon \ll 1$, a semi-classical calculation gives

$$
\left|t-t_{0}\right| \lesssim \frac{10(\xi / b)^{3}}{N^{1 / 2}} \ln \left(N b^{2} / \xi^{2}\right),
$$

a quantity checked to be $\ll 1$ in what follows.

We now study the experimental feasibility. An axial Gaussian laser beam confines $N \simeq 100$ atoms of ${ }^{7} \mathrm{Li}$ in the $y-z$ plane, with a resulting transverse harmonic oscillator length $a_{\perp}=\left(\hbar / m \omega_{\perp}\right)^{1 / 2} \simeq 0.54 \mu \mathrm{m}$, where $\omega_{\perp} \simeq 2 \pi \times 4.8 \mathrm{KHz}$ is the transverse oscillation frequency. In this optical wave guide, the interacting gas has a one dimensional character if $2 \xi \gg a_{\perp}$. In order to make cooling of the gas not too challenging, we take a not too large soliton length $\xi \simeq 0.9 \mu \mathrm{m}$; the resulting $3 \mathrm{D}$ scattering length, $a \simeq-a_{\perp}^{2} /(2 N \xi) \simeq-1.72 \mathrm{~nm}$ is in the interval of values $(-\infty,-1.5 \mathrm{~nm})$ accessible with the Feshbach resonance [1]. Initially the gas is also harmonically trapped along $x$ with an oscillation frequency $\omega$. The gas is assumed to be cooled to the temperature $T=0.45 \mathrm{nK}$ [6]. This axial trap is so weak that it very weakly affects the internal solitonic variables, $\hbar \omega<|\mu| / 10$, but it is strong enough that the center of mass of the gas, still separable in a harmonic trap, has a negligible probability $\exp \left(-\hbar \omega / k_{B} T\right)<1 / 10$ to be in an excited state. These two constraints impose the weak value $\omega \simeq 2 \pi \times 23.5 \mathrm{~Hz}$. They also imply $|\mu| / k_{B} T \simeq 25$, so that the internal variables of the soliton are frozen in their ground state.

At $t=0$, the gas is launched with a total momentum $\hbar K_{0}$ such that

$$
\frac{\hbar^{2} K_{0}^{2}}{2 M}=\frac{|\mu|}{2} \simeq \frac{\hbar^{2}}{16 m \xi^{2}} .
$$


The corresponding velocity is $\hbar K_{0} / M \simeq 0.37 \mathrm{~mm} / \mathrm{s}$. Simultaneously the axial trap is switched off, to free the center of mass of the gas, with an initial wavepacket

$$
\Phi(X) \propto e^{i K_{0} X} e^{-\left(X-X_{0}\right)^{2}(\Delta K)^{2}} .
$$

With a sudden opening of the axial trap [18, $\hbar^{2}(\Delta K)^{2} / 2 M=\hbar \omega / 4$ and $\Delta K / K_{0} \simeq 0.22 \ll 1$ : This wavepacket is quasi-monochromatic. Smaller values of $\Delta K$ may be obtained by a more clever opening procedure of the trap, within times $\sim 1 / \omega[19]$. The wavepacket is then scattered on a broad Gaussian barrier centered in $x=0$ (here $X_{0}<0$ ), a beam-splitter, created by a laser beam of waist $b=5 \xi \gg \xi$ and of intensity adjusted to satisfy the half-transmission probability condition (11). In any realistic case, $\Delta K$ remains much larger than $\delta K$, so the wavepacket experiences a mere filtering in Fourier space, the components with $K>K_{0}$ being transmitted and the ones with $K<K_{0}$ being reflected [20]. As a consequence, the wavepacket also splits in real space in a transmitted part and a reflected part, that nicely separate since their mean velocity exceeds their spreading velocity: A mesoscopic Schrödinger cat is born.

How to prove this experimentally? The first step is to check the absence of fragmentation: A photo of the gas by absorption imaging should show, for any realization of the experiment, that all the particles are clustered in a single lump of size $\xi$, randomly situated to the left or to the right of the beam-splitter. The second step is to check that the two wavepackets are coherent, by recombining them and looking for interference fringes, with a fringe spacing $\pi / K_{0}$. The recombination of the two wavepackets is obtained by their total reflection on mirrors, produced by two Gaussian laser beams centered in $x=L / 2$ and $x=-L / 2, L \gg 1 / \Delta K$, with the same waist as the beam splitter but with a higher intensity (say, twice as high). The reflected wavepackets interfere around $x=0$, the beam splitter being switched off [21].

We have studied the proposed experiment by a numerical solution of Schrödinger's equation for the center of mass wavefunction, with the initial condition (16) and the same approximate effective Hamiltonian as in scattering theory:

$$
i \hbar \partial_{t} \Phi(X, t)=\left[-\frac{\hbar^{2}}{2 M} \partial_{X}^{2}+\bar{V}(X, t)\right] \Phi(X, t)
$$

The center of mass probability distribution $|\Phi(X, t)|^{2}$ is plotted at key times in Fig,1. To quantify the contrast of the interference fringes, we also plotted the modulus of its Fourier transform, $s(Q, t)=\int_{-\infty}^{+\infty} d X e^{-i Q X}|\Phi(X, t)|^{2}$. When the two wavepackets overlap, sharp peaks in $|s(Q)|$ indeed form in $Q \simeq \pm 2 K_{0}$, with a contrast $|s| \simeq 0.32$. This is a high value, as the ideal case of two overlapping plane waves $\Phi(X) \propto e^{i K_{0} X}+e^{-i K_{0} X}$ gives $1 / 2$.

The high contrast interference fringes in Fig 1 are however for the center of mass probability distribution, not
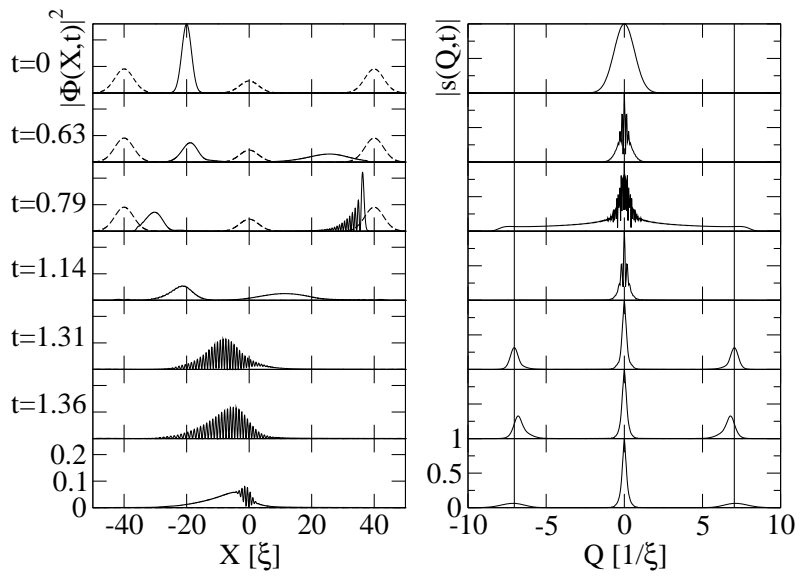

FIG. 1: Evolution of the center of mass wavefunction of a solitonic gas with $N=99$ atoms, by integration of (17) for the initial condition (16), with $\Delta K \simeq 0.093 K_{0}, X_{0}=-15 \xi$. Left panel: $|\Phi(X, t)|^{2}$ (solid lines), effective potential $\bar{V}(X, t)$ (dashed lines). Right panel: modulus of the Fourier transform $s(Q, t)$ of $|\Phi(X, t)|^{2}$; the vertical lines are in $Q= \pm 2 K_{0}$. The time is in units of $M L / \hbar K_{0}$. At $t=0.63$ the state is a cat. At $t=1.31$ the two reflected cat components strongly interfere, two narrow peaks of height 0.315 emerge on $|s(Q)|$ in $Q=$ $\pm 2 K_{0}$. Maximal interference occurs at $t=1.36$. The last line is an average over a Poisson distribution for $N$, with a mean value $\bar{N}=99$; the peak height in $|s(Q)|$ is reduced to 0.062 .

for the atomic density, which raises the question of their observability by usual fluorescence imaging. The mean atomic density $\rho(x)$ is the convolution of $|\Phi(X)|^{2}$ with the internal soliton density $\rho(x \mid 0)$; since the soliton size $\xi$ is as large as the fringe spacing $\pi / K_{0}$, one finds that the contrast of the fringes in $\rho(x)$ is several orders of magnitude smaller than in $|\Phi(X)|^{2}$. This problem can be solved by increasing, just before imaging, the intensity of the laser producing the transverse trapping by a factor about 21, which reduces the transverse harmonic oscillator length to $\tilde{a}_{\perp}=0.25 \mu \mathrm{m}$ and brings the soliton close to its collapse threshold $N|a| / \tilde{a}_{\perp} \simeq 0.67$ [22]. Furthermore fluorescence imaging can be optimized to measure directly the quantity $\left|s\left(2 K_{0}\right)\right|$, by exciting the gas with a laser standing wave along $x$, produced by the superposition of two laser waves of wavevectors $\vec{k}_{ \pm}=\left( \pm k_{x}, k_{y}, 0\right)$ at some angle with the $x$ axis such that $\vec{k}_{+}-\vec{k}_{-}=2 K_{0} \vec{e}_{x}$. The resulting fluorescence rate in direction $\vec{n}$ per unit of solid angle is given in the Born approximation by $d \Gamma / d \Omega \propto\left\langle\left|\sum_{i=1}^{N} e^{-i k \vec{n} \cdot \vec{r}_{i}} e\left(\vec{r}_{i}\right)\right|^{2}\right\rangle$, with validity conditions discussed in 23. Here $e(\vec{r})$ is the laser electric field. The emission rate $\Gamma_{\Omega}$ of photons in the solid angle $\Omega$ of the detection lens is an oscillating function of the location of the antinodes of the laser standing wave with respect to the interference pattern in $|\Phi(X)|^{2}$, with a contrast

$$
\frac{\Gamma_{\Omega}^{\max }-\Gamma_{\Omega}^{\min }}{\Gamma_{\Omega}^{\max }+\Gamma_{\Omega}^{\min }}=\left|s\left(2 K_{0}\right)\right| S_{\mathrm{in}}(\Omega) .
$$


The reduction factor $S_{\text {in }}(\Omega)$ is a function of the $3 \mathrm{D}$ static structure factor of the soliton for fixed center of mass position, that we approximate with the $3 \mathrm{D}$ mean field theory. By using a lens of optical axis along $\vec{k}_{+}+\vec{k}_{-}$ with a numerical aperture 0.4 , one finds the remarkably high value $S_{\text {in }}(\Omega)=0.84$, thanks to a superradiant effect [23], which also concentrates $16 \%$ of the fluorescence in the $4 \%$ solid angle fraction collected by the lens.

It remains to check that decoherence is negligible during the transit time $t_{\text {trans }}=M L / \hbar K_{0} \lesssim 200 \mathrm{~ms}$ of the cat state in the interferometer. In cold atom experiments, the main source of decoherence is particle losses: A single loss event would destroy the cat, since it "measures" the positions of one or several atoms and localizes the center of mass of the gas within the soliton size $\xi$. The usual loss rate formula for $m$-body loss is $d N / d t=-K_{m} \int d^{3} r n^{m}(\vec{r})$; here one should take for $n$ the $3 \mathrm{D}$ density profile for a fixed center of mass position, that we approximate with the mean field theory. For onebody losses due to collisions with the background gas, one should have a loss probability $K_{1} N t_{\text {trans }}<1 / 10$, which imposes the reasonable lifetime $K_{1}^{-1}>200$ s. For threebody losses due to formation of deeply bound dimers, the loss constant $K_{3}$ for ${ }^{7} \mathrm{Li}$ at the considered magnetic field $B$ is not known. Since $|a|$ is smaller than the Van der Waals length $3 \mathrm{~nm}$, as it is for $B=0$, we use the $B=0$ prediction of 24], applying the factor 6 reduction for a condensate, $K_{3} \approx 3 \times 10^{-41} \mathrm{~m}^{6} / \mathrm{s}$, which leads to a negligible loss event probability $\frac{1}{3}|d N / d t| t_{\text {trans }} \approx 0.03$.

In present experiments the number of atoms $N$ fluctuates from one realization to the other around the desired mean value $\bar{N}$. Since the launch velocity $\hbar K_{0} / M$ is fixed, $K_{0}$ is proportional to $N$ and also fluctuates [25]. A first side effect is that the half-transmission probability condition may be violated away from $N=\bar{N}$; fortunately this is not the case for a broad barrier $b \gg \xi$, since both terms of (11) are proportional to $N$. A second side effect is that the fringe spacing $\pi / K_{0}$ will fluctuate, which will blur the fringes. A simple way to estimate this is to assume that $|\Phi(X)|^{2} \propto\left|e^{i K_{0} X}+e^{-i K_{0} X}\right|^{2} e^{-X^{2} / 2 \sigma^{2}}$ at the overlap time. Averaging over a Poisson distribution for $N$ with $\sigma$ and $K_{0} / N$ fixed leads to, for $|X| \ll \pi \bar{N} / \bar{K}_{0}$ :

$$
\left\langle|\Phi(X)|^{2}\right\rangle \simeq \frac{e^{-X^{2} / 2 \sigma^{2}}}{(2 \pi)^{1 / 2} \sigma}\left[1+e^{-X^{2} / 2 \sigma_{c}^{2}} \cos \left(2 \bar{K}_{0} X\right)\right] .
$$

The fringes persist around the origin over a distance $\sigma_{c}=$ $\bar{N}^{1 / 2} /\left(2 \bar{K}_{0}\right)=\sqrt{2} \xi \cdot\left|s\left(2 \bar{K}_{0}\right)\right|$ is then reduced by a factor $\sigma_{c} /\left(\sigma^{2}+\sigma_{c}^{2}\right)^{1 / 2}$. Estimating $\sigma$ from Fig 1 leads to a reduction factor 5 close to the numerical one (see Fig 1).

In conclusion, we propose to produce a coherently bilocalized gas by scattering an atomic quantum soliton on a barrier. We have performed a detailed analysis of this idea, which raises challenging experimental aspects of preparation and detection, but also non trivial theoretical aspects since this is a many-body problem. We find that a gas with $N \simeq 100{ }^{7} \mathrm{Li}$ atoms can be prepared in a coherent superposition of being at two different locations separated by $\sim 100 \mu \mathrm{m}$, and that this can be proved by an interferometric measurement.

One of us (C.W.) acknowledges financial support from the European Union (contract MEIF-CT-2006-038407). We acknowledge useful discussions with A. Sinatra, C. Salomon. Our group is a member of IFRAF.

[1] L. Khaykovich et al., Science 296, 1290 (2002).

[2] K. Strecker et al., Nature 417, 150 (2002).

[3] C. Lee, J. Brand, Europhys. Lett. 73, 321 (2006); I. Mazets, G. Kurizki, Europhys. Lett. 76, 196 (2006).

[4] B. Lamine et al., Phys. Rev. Lett. 96, 050405 (2006).

[5] K. Hornberger et al., Phys. Rev. Lett. 90, 160401 (2003); L. Hackermueller et al., Nature 427, 711 (2004).

[6] A. Leanhardt et al., Science 301, 1513 (2003).

[7] M. Greiner et al., Nature 419, 51 (2002).

[8] C. Monroe et al., Science 272, 1131 (1996).

[9] M. Brune et al., Phys. Rev. Lett. 77, 4887 (1996).

[10] Y. Castin, J. Dalibard, Phys. Rev. A 55, 4330 (1997); J. Ruostekoski et al., Phys. Rev. A 57, 511 (1998); I. Cirac et al., Phys. Rev. A 57, 1208 (1998); Y. Castin, §8.2.3, in "Coherent atomic matter waves", Les Houches session LXXII, EDP Sciences \& Springer-Verlag (2001); J. Dunningham, K. Burnett, J. Mod. Opt. 48, 1837 (2001); A. Montina, F. Arecchi, Phys. Rev. A 66, 013605 (2002); A. Micheli et al., Phys. Rev. A 67, 013607 (2003); N. Teichmann, C. Weiss, EPL 78, 10009 (2007).

[11] Y. Castin, C. Herzog, Comptes Rendus de l'Académie des Sciences de Paris, vol. 2, series IV, p. 419 (2001).

[12] J.B. Mc Guire, J. Math. Phys. 5, 622 (1964).

[13] C. Cohen-Tannoudji, J. Dupont-Roc, G. Grynberg, in "Processus d'interaction entre photons et atomes", section III, InterEditions \& Editions du CNRS (Paris, 1988).

[14] F. Calogero, A. Degasparis, Phys. Rev. A 11, 265 (1975).

[15] This amounts to calculating for large $N$ the first "beyond mean field" correction for the static structure factor $S(x, y \mid X)$ with fixed center of mass position $X$. The integral of $S(x, y \mid X)$ over $X$ is the structure factor $S(x, y)$ of [16] so our large $N$ expansion implies the one of [16].

[16] P. Calabrese, J.-S. Caux, J. Stat. Mech. P08032 (2007).

[17] M. Berry, K. E. Mount, Rep. Prog. Phys. 35, 315 (1972).

[18] Up to order $N$, this creates a number $N_{\text {ex }}=\alpha N(\hbar \omega / \mu)^{4}$ of internal soliton excitations, with $\alpha=\left[\pi^{2}\left(\pi^{2}+25\right) / 30+\right.$ $\zeta(5)+5 \zeta(3)] / 128 \simeq 0.1447$. Here $N_{\text {ex }} \ll 1$.

[19] M. Morinaga et al., Phys. Rev. Lett. 83, 4037 (1999).

[20] This splitting by filtering is more robust experimentally than macroscopic quantum tunneling: Fluctuations of the laser intensity producing the barrier should only be such that resulting fluctuations of $K_{0}$ in (11) are $\ll \Delta K$.

[21] In a ring geometry, recombination is provided for free by time evolution, and our simulation results are similar.

[22] A. Gammal, L. Tomio, T. Frederico, Phys. Rev. A 66, 043619 (2002).

[23] J. Javanainen, Phys. Rev. Lett. 75, 1927 (1995).

[24] A. Moerdijk, H. Boesten, B. Verhaar, Phys. Rev. A 53, 916 (1996).

[25] $\Delta K$ also fluctuates, as $N^{1 / 2}$, since $\hbar^{2}(\Delta K)^{2} / 2 M \propto \hbar \omega$. 